\title{
NOETHERIAN INTERSECTIONS OF INTEGRAL DOMAINS
}

\author{
BY \\ WILLIAM HEINZER AND JACK OHM( $\left.{ }^{(}\right)$
}

\begin{abstract}
Let $D<R$ be integral domains having the same quotient field $K$ and suppose that there exists a family $\left\{V_{i}\right\}_{i \in I}$ of 1-dim quasi-local domains having quotient field $K$ such that $D=R \cap\left\{V_{i} \mid i \in I\right\}$. The goal of this paper is to find conditions on $R$ and the $V_{i}$ in order for $D$ to be noetherian and, conversely, conditions on $D$ in order for $R$ and the $V_{i}$ to be noetherian. An important motivating case is when the set $\left\{V_{i}\right\}$ consists of a single element $V$ and $V$ is a valuation ring. It is shown, for example, in this case that (i) if $V$ is centered on a finitely generated ideal of $D$, then $V$ is noetherian and (ii) if $V$ is centered on a maximal ideal of $D$, then $D$ is noetherian if and only if $R$ and $V$ are noetherian.
\end{abstract}

Our terminology is that of Zariski-Samuel [15] and Bourbaki [2]. All rings considered are commutative rings with identity, and except for several propositions concerning flat ring extensions, we deal only with integral domains. If $S$ and $T$ are sets, we use $S \backslash T$ to denote $\{s \in S \mid s \notin T\}$; and if $V$ is a valuation ring, we use $V(-)$ to denote the value of - with respect to an associated valuation. If $D$ is a subring of a ring $R$ and $P$ is a prime ideal of $D$, then we write $R_{P}$ for the quotient ring of $R$ with respect to the multiplicative system $D \backslash P$. We use $\bar{R}$ to denote the integral closure of an integral domain $R$; and finally, $\subset$ denotes containment and $<$ strict containment.

1. $R$-Krull domains. In proving the main theorems of this paper, we found that we were frequently using techniques that resembled those encountered in dealing with Krull rings, and toward the end of our work it became clear to us that a natural setting for our study is one that generalizes the language and ideas of classical Krull ring theory. Thus, the concept of an $R$-Krull domain will play a central role in our development, and we shall carry over to these rings a surprisingly large number of results, suitably generalized, from the theory of Krull rings.

We begin by fixing some notation and terminology which will remain in effect throughout the paper. $R$ and $D$ will always denote integral domains with the same

Presented to the Society, January 24, 1969 under the title Noetherian and non-Noetherian commutative rings; received by the editors October 12, 1968 and, in revised form, February 2, 1971.

AMS 1970 subject classifications. Primary 13E05, 13F05, 13B20.

Key words and phrases. 1-dim quasi-local integral domain, flat ring extension, Krull domain, essential valuation ring.

( $\left.{ }^{1}\right)$ The authors received partial support from the National Science Foundation during the writing of this paper. During completion of the paper the first named author was supported as an NRC-ONR Research Associate. 
quotient field $K$. A set of domains $\left\{D_{i}\right\}_{i \in I}$ with the same quotient field $K$ will be said to have $F C$ ( finiteness condition) if $0 \neq \xi \in K$ implies $\xi$ is a unit of $D_{i}$ for all but finitely many $i$, which is equivalent to the assertion that $0 \neq d \in D$ implies $d$ is a unit of $D_{i}$ for all but finitely many $i$. A nonempty set $\left\{V_{i}\right\}_{i \in I}$ of 1-dim quasi-local domains with quotient field $K$ will be called a set of $R$-representatives for $D$ if $\left\{V_{i}\right\}_{i \in I}$ has FC, $R \nsubseteq \bigcap_{i \in I} V_{i}$, and $D=R \cap\left(\bigcap_{i \in I} V_{i}\right)$. Note that when such a set exists, we must have $D<R$. If $\left\{V_{i}\right\}_{i \in I}$ is a set of $R$-representatives for $D$, we use $P_{i}$ to denote the center of $V_{i}$ on $D$, i.e. $P_{i}$ is the intersection of the maximal ideal of $V_{i}$ with $D . V_{i}$ is said to be irredundant (in the intersection $D=R \cap\left(\bigcap_{i \in I} V_{i}\right)$ ) if $R \cap\left(\bigcap_{j \neq i} V_{j}\right) \notin V_{i}$.

A valuation ring is called rational if its associated value group is order isomorphic to an additive subgroup of the rational numbers. A set $\left\{V_{i}\right\}_{i \in I}$ of $R$-representatives for $D$ will be called a set of rational R-representatives for $D$ if every $V_{i}, i \in I$, is a rational valuation ring; and $D$ will be called an $R$-Krull domain if there exists a set of rational $R$-representatives for $D$. Thus, an $R$-Krull domain is a domain $D$ having the same quotient field $K$ as $R$ and such that there exists a set $\left\{V_{i}\right\}_{i \in I}$ of rational valuation rings with quotient field $K$ such that $\left\{V_{i}\right\}_{i \in I}$ has $\mathrm{FC}, R \notin \bigcap_{i \in I} V_{i}$, and $D=R \cap\left(\bigcap_{i \in I} V_{i}\right)$.

The concept of an $R$-Krull domain is a continuation of the line of thought begun in $[12, \S 5]$. When $R=K$ and each $V_{i}$ is noetherian, then the notion of $R$-Krull domain coincides with that of Krull ring. Our goal in this section is to find conditions on $R$ and the $V_{i}$ in order for $D$ to be noetherian and conversely conditions on $D$ in order for $R$ and the $V_{i}$ to be noetherian. We begin by generalizing some well-known results on Krull rings.

1.1. Lemma. Let $\left\{D_{\alpha}\right\}$ be a set of overrings contained in $K$ of the integral domain $D$, and let $S$ be a multiplicative system of $D$. If $\left\{D_{\alpha}\right\}$ has $F C$ and $D=\bigcap_{\alpha} D_{\alpha}$, then $D_{S}=\bigcap_{\alpha}\left(D_{\alpha}\right)_{S}$. In particular, if $\left\{V_{i}\right\}$ is a set of $R$-representatives for $D$, then $D_{S}$ $=R_{S} \cap\left(\bigcap_{j \in J} V_{j}\right)$, where $\left\{V_{j}\right\}_{j \in J}$ is the set of $V_{i}$ such that $P_{i} \cap S=\varnothing$.

Proof. Let $\xi \in \bigcap\left(D_{\alpha}\right)_{S}$. By the FC, $\xi \notin D_{\alpha}$ for at most finitely many $\alpha$, say $\xi \notin D_{1}, \ldots, D_{n}$. There exists $s_{i} \in S$ such that $s_{i} \xi \in D_{i}, i=1, \ldots, n$; so if $s=s_{1} \cdots s_{n}$, then $s \xi \in D$ and $\xi \in D_{S}$. For the second assertion, observe that $\left(V_{i}\right)_{S}=K$ if $P_{i} \cap S$ $\neq \varnothing$.

1.2. Corollary. Let $\left\{V_{i}\right\}_{i \in I}$ be a set of $R$-representatives for $D$. If $P$ is a prime ideal of $D$ such that $R \nsubseteq D_{P}$, then $P_{i} \subset P$ for some $i \in I$.

Proof. Take $S=D \backslash P$ in 1.1 .

The next lemma plays an important role in what follows. Its proof is basically that of $[12$, Theorem 5.1, p. 330].

1.3. LEMMA. If $D, R$ are domains with quotient field $K$ and $V$ is a rational valuation ring with quotient field $K$ such that $R \nsubseteq V$ and $D=R \cap V$, then $V=D_{P}$, where $P$ is the center of $V$ on $D$. 
Proof. $D_{P} \subset V$ is immediate, so we shall concern ourselves with the reverse inclusion. If $\xi \in V$, we must show that there exists $d \in D \backslash P$ such that $d \xi \in R$. For this it is sufficient to exhibit an element $d \in R$ such that $V(d)=0$ and $d \xi \in R$. Since $V$ and $D$ have the same quotient field, $\xi=a / b, a, b \in D$; and since $R \notin V$, there exists $r \in R$ such that $V(r)<0$. If $b \notin P$, then $b$ is the required element. On the other hand, if $V(b)>0$, then since $V$ is a rational valuation ring, there exist integers $m, n \geqq 1$ such that $V\left(r^{n} b^{m}\right)=0$; thus, $d=r^{n} b^{m}$ is the required element. Q.E.D.

The example of [12, Example 5.3, p. 330] shows that 1.3 is no longer valid if one merely assumes that $V$ is a rank 1 valuation ring rather than a rational valuation ring.

1.4. Proposition. Let $D$ be an $R$-Krull domain and let $\left\{V_{i}\right\}_{i \in I}$ be a set of rational $R$-representatives for $D$. Then $\mathscr{E}=\left\{D_{P} \mid R \notin D_{P}\right.$ and $D_{P}=V_{i}$ for some $\left.i\right\}$ is also a set of rational $R$-representatives for $D$.

Proof. Since any $D_{P} \in \mathscr{E}$ equals some $V_{i}$, certainly $\mathscr{E}$ has $\mathrm{FC}$ and each $D_{P} \in \mathscr{E}$ is a rational valuation ring. It therefore remains to see that $D=R \cap\left\{D_{P} \mid D_{P} \in \mathscr{E}\right\}$. $C$ is clear, so suppose $x$ is in the right-hand side of the equality but is not in $D$. Then there exists at least one $V_{j}$ such that $x \notin V_{j}$, and by the FC there exist at most finitely many such $V_{j}$; call these $V_{1}, \ldots, V_{n}$. Then $D=R^{\prime} \cap V_{1} \cap \cdots \cap V_{n}$, where $R^{\prime}=R \cap\left(\bigcap V_{i}\right), i \in I \backslash\{1, \ldots, n\} . D \neq R^{\prime}$ since $x \in R^{\prime} \backslash D$, so we may assume that $V_{1}$ is irredundant in this intersection. It follows by 1.3 that $V_{1}$ is a quotient ring of $D$. Therefore $V_{1} \in \mathscr{E}$, and hence $x \in V_{1}$, a contradiction.

1.5. TheOREM. Let $D$ be an $R$-Krull domain, let $\left\{V_{i}\right\}_{i \in I}$ be a set of rational $R$ representatives for $D$, and let $P_{i}$ denote the center of $V_{i}$ on $D$. If $P$ is a prime ideal of $D$ such that $R \notin D_{P}$, then the following are equivalent:

(i) $P_{i} \subset P$ implies $P_{i}=P$, for all $i \in I$.

(ii) There exists $j \in I$ such that $V_{j}=D_{P}$.

(iii) There exists $j \in I$ such that $P_{j}=P$ and $V_{j}$ is irredundant.

(iv) $P=P_{j}$ for some $j \in I, P$ is minimal, and no prime ideal of $R$ lies over $P$.

(v) $P$ is minimal.

Proof. (i) $\Rightarrow$ (ii). By $1.1, D_{P}=R_{P} \cap\left(\bigcap_{j \in J} V_{j}\right)$, where $\left\{V_{j}\right\}_{j \in J}$ are those $V_{i}$ having center $P_{i} \subset P . D_{P} \neq R_{P}$, since otherwise $R \subset D_{P}$; so $J \neq \varnothing . J$ is then finite by the FC, since $P_{i} \subset P$ implies $P_{i}=P$ by (i). Therefore we can delete finitely many elements from $\left\{V_{j}\right\}_{j \in J}$ to get an irredundant intersection $D_{P}=R_{P} \cap V_{1} \cap \cdots \cap V_{t}$. By 1.3, $V_{1}=D_{P}$.

(ii) $\Rightarrow$ (iii). $V_{j}=D_{P}$ implies $P$ is minimal and $V_{j}$ is the only $V_{i}$ centered on $P$. If $V_{j}$ is redundant, then $D=R \cap\left(\bigcap_{i \neq j} V_{i}\right)$. But then (i) $\Rightarrow$ (ii) applied to the set of $R$-representatives $\left\{V_{i}\right\}_{i \neq j}$ implies that there exists $V_{i}, i \neq j$, such that $V_{i}$ is centered on $P$, a contradiction.

(iii) $\Rightarrow$ (iv). $V_{j}$ is irredundant implies $V_{j}=D_{P_{j}}$ by 1.3 ; and since $V_{j}$ is 1 -dim, it follows that $P_{j}$ is minimal. If now $Q$ is a prime ideal of $R$ such that $Q \cap D=P$, then 
$V_{j}=D_{P} \subset R_{Q}$. But $V_{j}$ is a rank 1 valuation ring, so then $V_{j}=R_{Q}$; and hence $R \subset R_{Q}$ $=V_{j}=D_{P}$, a contradiction to our hypothesis.

(iv) $\Rightarrow$ (v) $\Rightarrow$ (i). Trivial.

1.6. Application. Let $D$ be an $R$-Krull domain and let $\left\{V_{i}\right\}_{i \in I}$ be a set of rational $R$-representatives for $D$. (ii) $\Leftrightarrow$ (iii) $\Leftrightarrow$ (v) of 1.5 shows that \{irredundant $V_{i}$ 's $=\left\{V_{i} \mid R \notin V_{i}\right.$ and $\left.V_{i}=D_{P_{i}}\right\}=\left\{D_{P} \mid P\right.$ is minimal and $\left.R \notin D_{P}\right\}$. We shall denote this set by $\mathscr{E}$. Note that the characterization $\mathscr{E}=\left\{D_{P} \mid P\right.$ is minimal and $\left.R \notin D_{P}\right\}$ shows that $\mathscr{E}$ is independent of the choice of the set of rational $R$-representatives $\left\{V_{i}\right\}$. Also, we have already seen in 1.4 that $\mathscr{E}$ is itself a set of rational $R$-representatives for $D$. We shall call $\mathscr{E}$ the set of essential $R$-representatives for $D$, and we shall call the centers on $D$ of the elements of $\mathscr{E}$ the essential primes of $D$. Thus, $\mathscr{E}$ is a set of irredundant rational $R$-representatives for $D$; and given any set of rational $R$ representatives for $D$, the subset of irredundant elements is exactly $\mathscr{E}$. (If one merely assumes that the $V_{i}$ are rank 1 valuation rings rather than rational valuation rings, the example of [12, Example 5.3, p. 330] shows that not every irredundant $V_{\iota}$ need be in $\mathscr{E}$.)

We shall frequently use in what follows a couple of facts involving flatness, one of which is the following criterion [14, Theorem 2, p. 795]. Let $D \subset R$ be domains with the same quotient field. Then $R$ is flat over $D$ if and only if for any prime ideal $P$ of $D$, either $R \subset D_{P}$ or $P R=R$. The second fact is that if $R$ is flat over $D$ and contained in the quotient field of $D$, then $D$ is noetherian implies that $R$ is noetherian [14, Corollary, p. 796].

We are now ready to examine the noetherian properties of $R$-Krull domains. Theorem 1.10 constitutes part of the main result of this section. Its proof requires the corollary to the next proposition.

1.7. Proposition. Let $\mathscr{R}$ be a ring and let $\left\{\mathscr{R}_{\alpha}\right\}$ be a set of flat $\mathscr{R}$-algebras (or $\mathscr{R}$-modules). Let $A$ be an ideal of $\mathscr{R}$ such that for each maximal ideal $M \supset A$ of $\mathscr{R}$ there exists an $\mathscr{R}_{\alpha}$ such that $M \mathscr{R}_{\alpha} \neq \mathscr{R}_{\alpha}$. If $A^{\prime}$ is an ideal of $\mathscr{R}$ such that $A^{\prime} \mathscr{R}_{\alpha} \subset A \mathscr{R}_{\alpha}$ for each $\alpha$, then $A^{\prime} \subset A$. Moreover, if for each $\alpha, A \mathscr{R}_{\alpha}$ is finitely generated as an ideal of $\mathscr{R}_{\alpha}$ (or finitely generated as an $\mathscr{R}$-module) and if there exists a finitely generated ideal $B \subset A$ such that $B \mathscr{R}_{\alpha} \neq A \mathscr{R}_{\alpha}$ for at most a finite number of $\alpha$, then $A$ is finitely generated.

Proof. If $b \in A^{\prime}$, then $(A: b) \mathscr{R}_{\alpha}=A \mathscr{R}_{\alpha}: b$ (where $A \mathscr{R}_{\alpha}: b=\left\{y \in \mathscr{R}_{\alpha} \mid b y \in A \mathscr{R}_{\alpha}\right\}$ ), [2a, Exercise 22, p. 65] or [11, 18.10, p. 58]. Since $b \mathscr{R}_{\alpha} \subset A^{\prime} \mathscr{R}_{\alpha} \subset A \mathscr{R}_{\alpha}$ we have $(A: b) \mathscr{R}_{\alpha}=\mathscr{R}_{\alpha}$ for each $\alpha$. Thus $A: b$ is contained in no maximal ideal of $\mathscr{R}$, so $A: b$ $=\mathscr{R}$, i.e. $b \in A$ and $A^{\prime} \subset A$. Assume now that $A \mathscr{R}_{\alpha}$ is finitely generated for each $\alpha$ and that $B \subset A, B$ finitely generated, is such that $B \mathscr{R}_{\alpha}=A \mathscr{R}_{\alpha}$ for all but a finite number of $\alpha$, say $\alpha=1,2, \ldots, n$. We can choose $A_{i} \subset A, A_{i}$ finitely generated, such that $A_{i} \mathscr{R}_{i}=A \mathscr{R}_{i}$. Then $B^{\prime}=B+A_{1}+\cdots+A_{n}$ is such that $A \mathscr{R}_{\alpha} \subset B^{\prime} \mathscr{R}_{\alpha}$ for each $\alpha$. Thus $A=B^{\prime}$ and $A$ is finitely generated. Q.E.D.

The following corollary includes the well-known statement that a quasi-semilocal domain which is locally noetherian is noetherian [15, II, Lemma 2, p. 327]. 
1.8. Corollary. Let $\mathscr{R}$ be a ring and let $\left\{\mathscr{R}_{\alpha}\right\}$ be a set of flat $\mathscr{R}$-algebras (or $\mathscr{R}$-modules) such that for each proper ideal $A$ of $\mathscr{R}, A \mathscr{R}_{\alpha} \neq \mathscr{R}_{\alpha}$ for at least one and at most a finite number of $\mathscr{R}_{\alpha}$. If each $\mathscr{R}_{\alpha}$ is a noetherian ring (or a noetherian $\mathscr{R}$ module), then $\mathscr{R}$ is noetherian.

For an incidental application of 1.8 , let us consider the following question: Let $\mathscr{R}$ be a noetherian ring and $\mathscr{R}_{1}, \ldots, \mathscr{R}_{n}$ be a set of flat overrings of $\mathscr{R}$ which are contained in the total quotient ring of $\mathscr{R}$. Is $\mathscr{R}^{\prime}=\bigcap_{i=1}^{n} \mathscr{R}_{i}$ necessarily noetherian? The answer is "no" in general. In fact [7, Example 2.10] gives an example of a noetherian domain $D$ having quotient rings $D_{1}, D_{2}$ such that $D_{1} \cap D_{2}$ is not noetherian. However, the following is true.

1.9. Proposition. If $\mathscr{R}$ is a noetherian ring and $\mathscr{R}_{1}, \ldots, \mathscr{R}_{n}$ are quasi-semilocal flat overrings of $\mathscr{R}$ which are contained in the total quotient ring of $\mathscr{R}$, then $\mathscr{R}^{\prime}=\bigcap \mathscr{R}_{i}$ is noetherian.

Proof. The $\mathscr{R}_{i}$ are flat over $\mathscr{R}$ and hence also are noetherian (see, for example, [1a, Corollary 1, p. 804]). Since every nonunit of $\mathscr{R}^{\prime}$ is also a nonunit in some $\mathscr{R}_{i}$, it follows that the set of nonunits of $\mathscr{R}^{\prime}$ is exactly the union of the finite set of contracted maximal ideals of the $\mathscr{R}_{i}$. Therefore every maximal ideal of $\mathscr{R}^{\prime}$ is the contraction of a maximal ideal of some $\mathscr{R}_{i}$. Finally, since the $\mathscr{R}_{i}$ are contained in the total quotient ring of $\mathscr{R}$ and are flat over $\mathscr{R}$, they are also flat over $\mathscr{R}^{\prime}[1 \mathrm{~b}$, Corollary 1, p. 803]. Thus, by $1.8, \mathscr{R}^{\prime}$ is noetherian. Q.E.D.

The next theorem is central to this paper.

1.10. Theorem. Let $D$ be an $R$-Krull domain, let $\left\{V_{i}\right\}_{i \in I}$ be a set of rational $R$ representatives for $D$, and suppose that the center on $D$ of every irredundant $V_{i}$ is maximal. Then $R$ is flat over $D$, and $D$ is noetherian if and only if $R$ and the irredundant $V_{i}^{\prime}$ 's are noetherian.

Proof. By 1.6 the irredundant $V_{i}$ are precisely the elements of $\mathscr{E}$, i.e. the essential $R$-representatives for $D$. Let $P$ be any prime ideal of $D$ such that $R \notin D_{P}$. Since the center on $D$ of any element of $\mathscr{E}$ is assumed maximal, 1.5(i) is satisfied by $P$; and hence by $1.5(\mathrm{iv}), P$ is itself the center of an element of $\mathscr{E}$ and therefore maximal, and no prime ideal of $R$ lies over $P$. This implies that $P R=R$. Thus, $R \notin D_{P}$ implies $P R=R$, which proves that $R$ is flat over $D$. It follows that $D$ is noetherian implies $R$ is noetherian.

We use 1.8 for the converse. As we have seen, for any prime ideal $P$ of $D$, either $R \subset D_{P}$, in which case $P R \neq R$, or $R \notin D_{P}$, in which case $P$ is the center of an element $V$ of $\mathscr{E}$ and $P V \neq V$. Thus, since $\mathscr{E}$ has FC and each element of $\mathscr{E}$ is a quotient ring of $D, 1.8$ applies. Q.E.D.

Note that another way of stating 1.10 is the following: Let $D$ be an $R$-Krull domain whose essential primes are maximal. Then $R$ is flat over $D$, and $D$ is noetherian if and only if $R$ is noetherian and the essential primes of $D$ are finitely generated. Moreover, when the essential primes of $D$ are maximal, $R$ is completely 
determined by these primes, for it follows from the flatness of $R[14, \mathrm{p} .795]$ that then $R=\bigcap\left\{D_{Q} \mid Q\right.$ a nonessential prime of $\left.D\right\}$.

We shall next examine a few examples to determine the bounds for possible generalizations of 1.10. A simple example in [3, p. 282] shows that there exist 1-dim local domains $R, V$ with $D=R \cap V$ being 1-dim quasi-local but not noetherian; so the hypothesis that the $V_{i}$ are rational valuation rings in 1.10 cannot be replaced by the hypothesis that the $V_{i}$ are 1-dim local. (The $D$ in this example is also integrally closed.)

The following modification of [12, Example 5.3, p. 330] shows that the assumption that the $V_{i}$ are rational valuation rings is also crucial for the flatness assertion of 1.10 .

1.11. Example of $D=R \cap V$ such that $V$ is a rank 1 valuation ring centered on a maximal ideal of $D$ and yet $R$ is not flat over $D$. Let $k$ be the rational numbers, let $x$ be an indeterminate, and let $V$ be the extension of the $p$-adic valuation $v$ of $k$ to $k(x)$ obtained by defining $V\left(a_{0}+a_{1} x+\cdots+a_{n} x^{n}\right)=\inf \left\{v\left(a_{i}\right)+i \pi\right\}, i=0, \ldots, n$. Let $D=k[x] \cap V$, let $M$ denote the center of $V$ on $D$, and let $P$ denote the center of the $x$-adic valuation ring of $k(x)$ on $D$. Then $P<M$, so there exists a rank 2 valuation ring $W$ of $k(x)$ such that $W \supset D$ and $W$ has prime ideals lying over $M$ and $P$. If now we let $R=k[x] \cap W$, then $D=R \cap V$.

It is shown in [12, p. 331] that $M$ is the only nonminimal prime ideal of $D$ and that any other prime ideal of $D$ is the contraction of a prime ideal of $k[x]$. Since $M$ is the center of $W$ on $D$, it follows that every prime ideal of $D$ is the contraction of a prime ideal of $R$. Thus, it will follow that $R$ is not flat over $D$ if we show that $D \neq R$. For sufficiently large $n, x / p^{n} \notin V$ and hence $\notin D$; but $W(x)$ is infinitely larger than $W(p)$, so $x / p^{n} \in W$ and hence $\in R=k[x] \cap W$. Q.E.D.

The following can happen if one omits the condition that the center of each irredundant $V_{i}$ be maximal in Theorem 1.10:

(i) $D$ may be noetherian, but $R$ may not be noetherian,

(ii) $R, V_{i}, i \in I$, may all be noetherian but $D$ may not be noetherian. (We show in 1.17 that when $D$ is noetherian the irredundant $V_{i}$ are always noetherian.) An example which shows (i) is constructed in [4, $\$ 2]$, where $R$ is an $A$-transform of a 3-dim normal affine ring $D$ and $\left\{V_{i}\right\}$ is a finite set of discrete rank 1 valuation rings. To see (ii), it suffices to have a 2-dim, quasi-local, nonnoetherian Krull domain $D$ having a minimal prime $P$ such that $P=\sqrt{ }(x)$ for some $x \in D$. For then take $R=D[1 / x]$ and $V=D_{P} . R$ is 1 -dim Krull and hence noetherian, and $D=R \cap V$ since $V$ is the only essential valuation ring of $D$ which does not contain $1 / x$. The existence of such a $D$ is proved in $[9$, p. 28].

The following corollary, whose proof is immediate, includes [6, Proposition 1] as a special case.

1.12. Corollary. Let $D$ be a Krull domain with the set of essential valuation rings $\mathscr{E}$, and let $\mathscr{M}$ be any subset of $\{V \in \mathscr{E} \mid$ the center of $V$ on $D$ is maximal $\}$. If $R=\bigcap\{V|V \in \mathscr{E}| \mathscr{M}\}$, then $R$ is noetherian if and only if $D$ is noetherian. 
We next prove a generalization of 1.12 . The generalization requires the following lemma which is taken from [13].

1.13. LEMMA. Let $R$ be an integral domain and let $\mathscr{P}$ be a set of minimal primes of $R$. If $\left\{R_{P} \mid P \in \mathscr{P}\right\}$ has $F C$ and $R=\bigcap\left\{R_{P} \mid P \in \mathscr{P}\right\}$, then $\mathscr{P}$ is the set of all minimal primes of $R$.

Proof. Let $Q$ be a minimal prime of $R$ and suppose that $Q \notin \mathscr{P}$. Choose $y \neq 0 \in Q$, and let $P_{1}, \ldots, P_{t}$ be the primes in $\mathscr{P}$ such that $y \in P_{i}(t \geqq 1$ since otherwise $\left.1 / y \in \cap R_{P}=R\right)$. Choose $x \in\left(P_{1} \cap \cdots \cap P_{t}\right) \backslash Q$.

Claim. $x^{n} / y \in \bigcap R_{P}=R$, for some $n$. For any $n, x^{n} / y \in R_{P}, P \neq P_{1}, \ldots, P_{t}$, since $1 / y \in R_{P}, P \neq P_{1}, \ldots, P_{t}$. Moreover, $R_{P_{i}}$ is 1 -dim quasi-local implies $x^{n_{i}} \in y R_{P_{i}}$ for some $n_{i}, i=1, \ldots, t$. Choose $n=\max \left\{n_{i}\right\}$, and then $x^{n} \in y R \subset Q$, a contradiction to the choice of $x$.

1.14. TheOREM. Let $D$ be a domain, and let $\mathscr{M}=\{$ minimal prime ideals of $D\}$. Assume $D=\bigcap\left\{D_{P} \mid P \in \mathscr{M}\right\}$, and that $\left\{D_{P} \mid P \in \mathscr{M}\right\}$ has FC. Let $\mathscr{N}$ be a subset of $\mathscr{M}$ such that every $P \in \mathscr{N}$ is maximal. Then $R=\bigcap\left\{D_{Q} \mid Q \in \mathscr{M} \backslash \mathscr{N}\right\}$ is flat over $D$.

Proof. By 1.13, the minimal prime ideals of $R$ are exactly $\left\{Q D_{Q} \cap R|Q \in \mathscr{M}| \mathcal{N}\right\}$. Therefore $P R=R$ for any $P \in \mathscr{N}$, for otherwise some minimal prime of $R$ would necessarily contract to $P$ by the maximality of $P$. If now $Q$ is any prime of $D$ such that $R \notin D_{Q}$, then $P \subset Q$ for some $P \in \mathscr{N}$, by 1.2. Therefore $P=Q$ and hence $Q R=R$, which proves that $R$ is flat over $D$.

1.15. Corollary. With the notation and hypotheses of $1.14, R$ and $\left\{D_{P} \mid P \in \mathscr{N}\right\}$ are noetherian if and only if $D$ is noetherian.

Proof. $R$ is flat over $D$, so $D$ is noetherian implies $R$ is noetherian. For the converse, observe that for any prime ideal $M$ of $D$, if $M R=R$, then $M \in \mathscr{N}$ by 1.2 ; and then apply 1.8. Q.E.D.

Our next theorem considerably sharpens 1.10 in the direction " $D$ is noetherian implies the irredundant $V_{i}$ are noetherian". It and 1.10 should be considered the principal results of this section.

1.16. Lemma. Let $A$ be a nonzero finitely generated ideal of an integral domain $D$ with quotient field $K$. Then $A: A=\{\xi \in K \mid \xi A \subset A\}$ is a ring integral over $D$.

Proof. It is immediate that $A: A$ is a ring. To see the integralness, we use a standard argument (see [15a, p. 216]). Let $A=\left(a_{1}, \ldots, a_{n}\right)$ and let $d \in A: A$. Then $d a_{i}=\sum_{j=1}^{n} y_{i j} a_{j}$, with $y_{i j} \in D$. Therefore $\operatorname{det}(d Z-Y)=0$, where $Z$ is the identity matrix and $Y=\left(y_{i j}\right)$; and this is the desired integral equation for $d$.

1.17. TheOREM. Let $D, R, V$ be domains with quotient field $K$, and suppose that $D=R \cap V=R \cap \bar{D}$, that $D \neq R$, and that $V$ is 1-dim quasi-local with center $P$ on $D$. If $P$ is finitely generated, then $V$ is a noetherian valuation ring. (Note that if $V$ is integrally closed, then $\bar{D} \subset V$, so $D=R \cap V=R \cap \bar{D}$.) 
Proof. By replacing $D, R$ by their quotient rings with respect to the multiplicative system $D \backslash P$, we are reduced (by 1.1) to considering the case where $D$ is quasi-local with maximal ideal $P$. Then either $P P^{-1}=D$ or $P P^{-1}=P$.

If $P P^{-1}=D$, then $P$ is invertible. Since $D$ is quasi-local, this implies that $P$ is principal [2a, Theorem 4, p. 148]. Also, since $P$ is contained in the height 1 maximal ideal of $V, \bigcap_{n=1}^{\infty} P^{n}=0$ [12, Corollary 1.4, p. 323]. These two properties imply that $D$ is a noetherian valuation ring ([2c, Proposition 9, p. 109]); so $D=V$, and we are done.

If, on the other hand, $P P^{-1}=P$, then $P^{-1}=P: P$. But $P: P$ is integral over $D$ by 1.16, and hence $P^{-1} \subset \bar{D}$. If now $Q \neq P$ is any other prime ideal of $D$, then $R \subset D_{Q}$ by 1.2. Therefore if $r \in R$ and if $\partial(r)=\{d \in D \mid d r \in D\}$, then $\partial(r) \notin Q$. Thus, $\sqrt{ } \partial(r)$ $=P$; so $P$ is finitely generated implies that $P^{n} \subset \partial(r)$ for some $n \geqq 1$. Hence $r P^{n} \subset D$ and $r \in P^{-n}$ (where by definition $P^{-n}=\left(P^{n}\right)^{-1}$ ). Choose $r \in R \backslash D$. Then there exists $n \geqq 1$ such that $r \in P^{-n}, \notin P^{-n+1}$. Then $r P^{n-1} \subset R$ and $r P^{n-1} \subset P^{-n} P^{n-1} \subset P^{-1} \subset \bar{D}$; so $r P^{n-1} \subset R \cap \bar{D}=D$, and hence $r \in P^{-n+1}$, a contradiction to the choice of $n$. Q.E.D.

1.18. Corollary. Suppose $D=R \cap\left\{V_{i} \mid i \in I\right\}$, where $\left\{V_{i}\right\}_{i \in I}$ is a set of 1-dim quasi-local integrally closed domains having FC. If each $V_{i}$ is centered on a finitely generated maximal ideal of $D$, then $D$ is noetherian if and only if $R$ is noetherian.

Proof. Apply 1.17 and 1.10 .

The condition $R \cap \bar{D}=D$ of 1.17 cannot be weakened to $R \cap \bar{V}$ is integral over $D$, as the following example shows.

1.19. Example of domains $R, V, D$ such that $D=R \cap V, R \notin V, V$ is 1-dim quasilocal and the center of $V$ on $D$ is maximal, yet $R, D$ are noetherian and $V$ is not. The $R$ and $D$ will, in fact, be 2-dim local domains with $R$ integral over $D$. Let $k_{0}<k$ be fields with $k$ a finite algebraic extension of $k_{0}$, and let $x, y$ be indeterminates over $k$; let $R=k[x, y]_{(x, y)}(=k+M$, where $M$ is the maximal ideal of $R)$; let $\bar{V}$ be the rank 1 valuation ring of $k(x, y)$, trivial on $k$, defined (using the procedure of [2c, p. 160]) by setting $\bar{V}(x)=1, \bar{V}(y)=\pi$; and let $V=k_{0}+N$, where $N$ is the maximal ideal of $\bar{V}$. Note that $\bar{V}=k+N$ and that $R \cap N=M$. Let $D=R \cap V$. Then $D=k_{0}+M$, so $R$ is a finite integral extension of $D$, and hence $D$ is noetherian by Eakin's theorem [3] (see [10] for a simplified proof of this theorem). On the other hand $V$ is not noetherian since $\bar{V}$ is not.

It is possible to construct a more complicated example than the above which has the additional property that $R \notin \bar{D}$.

As another application of 1.17 and 1.10 , we mention the following corollary, which includes a situation treated in [15, II, p. 328].

1.20. Corollary. Let $V$ be a rank 1 valuation ring and $R$ be a quasi-semilocal ring, let $D=R \cap V$, and suppose $D, R, V$ all have the same quotient field. If there exists an element $x$ which is a unit of $R$ and a nonunit of $V$, then $D$ is noetherian if and only if $V, R$ are noetherian. 
Proof. The existence of $x$ implies that $V$ is irredundant. Since every nonunit of $D$ is either a nonunit of $R$ or a nonunit of $V$, the set of nonunits of $D$ is precisely the union of the finite number of contracted maximal ideals from $R$ and $V$. Thus, $D$ is quasi-semilocal, and the condition on $x$ then implies that the center of $V$ on $D$ is maximal; so 1.17 and 1.10 apply. Q.E.D.

The converse to 1.17 is false; for it can be seen that the example of [12, Example 5.3 , p. 330] gives a domain $D$ which is an irredundant intersection of rank 1 valuation rings, all but one of which are noetherian and such that an infinite number of them have nonfinitely generated center. The existence of Krull rings having minimal primes which are not finitely generated (see for example, [4]) gives another source of counterexamples to the converse of 1.17 . However, if one imposes the further condition that the center of $V$ be maximal, then the converse is easily proved. Thus,

1.21. Proposition. Let $R, V, D, P$ be as in 1.17. If $P$ is maximal and $V$ is a noetherian valuation ring, then $P$ is finitely generated.

Proof. If $M \neq P$ is a maximal ideal of $D$, then $D_{M} \supset R$ by 1.2. Also, $P R=R$ and $V=D_{P}$ by 1.5 . Therefore there exists a finitely generated ideal $P_{0}$ of $D$ such that $P_{0} R=R=P R$ and $P_{0} V=P V$. Then $P_{0} D_{M}=P D_{M}$ for every maximal ideal of $D$, so $P_{0}=P$. Q.E.D.

It is perhaps worth noting that this proposition yields immediately (and hence provides a simple proof of) the main result, Theorem 3, p. 338, of [5], which asserts that if a domain $D$ has the property that every $D_{P}, P$ maximal, is a rank 1 discrete valuation ring and $D=\bigcap D_{P}$ is an irredundant intersection, then $D$ is noetherian.

Let $D$ be an integral domain with quotient field $K$. We remind the reader that an element $\xi \in K$ is called almost integral (or quasi-integral) over $D$ if there exists a nonzero $d \in D$ such that $d \xi^{i} \in D$ for all $i=1,2, \ldots$ An element which is integral over $D$ is almost integral over $D$, and the concepts coincide when $D$ is noetherian. (See for example, [2c, p. 20].)

1.22. Corollary (TO 1.17). Let $D \neq R$ be domains with quotient field $K$. If $W$ is a valuation ring of $K$ such that $D=R \cap W$ and such that $W$ is maximal with respect to this property (i.e. there does not exist a valuation ring $U$ of $K$ such that $U>W$ and $R \cap U=D$ ), then $D$ is noetherian implies $W$ is noetherian.

Proof. By 1.17, it suffices to see that $W$ has rank 1. If not, there exists a nontrivial valuation ring $W^{\prime}>W$. If $M^{\prime}$ is the maximal ideal of $W^{\prime}$, then $M^{\prime} \subset W$; also $M^{\prime} \cap D \neq 0$ since $D$ and $W^{\prime}$ have the same quotient field. Therefore, if $x \in W^{\prime} \cap R$ and $d \neq 0 \in M^{\prime} \cap D$, then $d x^{n} \in M^{\prime} \cap R \subset D, n=1,2, \ldots$ Thus, $x$ is almost integral over $D$; and since $D$ is noetherian, this implies $x$ is integral over $D$. Therefore $x \in W$, so $W^{\prime} \cap R=D$, a contradiction to the choice of $W$. Q.E.D.

The 1-dim assumption in 1.17 is essential, as is seen by the following example. Take $R=k[x, y, z, 1 / x]$, where $x, y, z$ are indeterminates over the field $k$. Let $V_{1}$ 
be the $x$-adic valuation ring of $k(x, y, z)$, and let $V$ be the composite of $V_{1}$ with some nondiscrete valuation ring containing $k[y, z]$ of the residue field $(\cong k(y, z))$ of $V_{1}$. Then $R \cap V=k[x, y, z]=D$ since $R \cap V_{1}=k[x, y, z]$, but the maximal ideal of $V$ is not finitely generated since $V$ has a nondiscrete homomorphic image.

We conclude this section with the following intrinsic characterization of $R$ Krull domains. It is modeled after the corresponding statement for Krull rings in $[11$, p. 115].

1.23. TheOREM. Let $D<R$ be integral domains having the same quotient field $K$. Then $D$ is an $R$-Krull domain if and only if

(i) if $P$ is a minimal prime of $D$ such that $R \notin D_{P}$, then $D_{P}$ is a rational valuation ring, and

(ii) if $x \in D, x \neq 0$, then $x D=(x R \cap D) \cap\left\{Q_{j} \mid j \in J\right\}$, where $\left\{Q_{j}\right\}_{j \in J}$ is a finite (possibly empty) family of primary ideals for minimal primes $P_{j}$ of $D$.

Proof. $(\Rightarrow)$ From 1.6, $\left\{D_{P} \mid P\right.$ is minimal and $\left.R \notin D_{P}\right\}$ is precisely the set $\mathscr{E}$ of essential $R$-representatives for $D$. Thus each such $D_{P}$ is a rational valuation ring and $D=R \cap\left\{D_{P} \mid D_{P} \in \mathscr{E}\right\}$. Hence if $x \in D, x \neq 0$, then $x D=x R \cap\left\{x D_{P} \mid D_{P} \in \mathscr{E}\right\}$. Since $\mathscr{E}$ has FC, $x D_{P} \neq D_{P}$ for only a finite number of the $D_{P} \in \mathscr{E}$, say $\left\{D_{P_{j}} \mid j \in J\right\}$. If $Q_{j}=x D_{P_{j}} \cap D$, then $Q_{j}$ is $P_{j}$-primary and $x D=(x R \cap D) \cap\left\{Q_{j} \mid j \in J\right\}$.

$(\Leftarrow)$ Let $\left\{P_{i} \mid i \in I\right\}$ be the set of minimal primes of $D$ such that $R \notin D_{P_{i}}$. By assumption each $D_{P_{i}}$ is a rational valuation ring. Also we have $D_{P_{i}}<R_{P_{i}}$. Hence $R_{P_{i}}=K$, i.e. each nonzero ideal of $R$ meets $D \backslash P_{i}$. If $x \in D, x \neq 0$, and $x D=$ $(x R \cap D) \cap\left\{Q_{j} \mid j \in J\right\} \subset P_{i}$, then we claim that $P_{i} \in\left\{P_{j} \mid j \in J\right\}$. For $x R \cap D \notin P_{i}$ implies that some $Q_{j} \subset P_{i}$. Thus the set $\left\{D_{P_{i}} \mid i \in I\right\}$ has FC. It only remains to show that $D=R \cap\left\{D_{P_{i}} \mid i \in I\right\}$. We note first that if a minimal prime $P_{j}$ of $D$ is such that $R \subset D_{P_{j}}$, then $x R \cap D \subset x D_{P_{j}} \cap D$. Hence we may assume that the minimal primes $P_{j}$ in (ii) are such that $R \notin D_{P_{j}}$. Thus for nonzero $x \in D$, we have from (ii) that $x D=(x R \cap D) \cap\left\{x D_{P_{i}} \mid i \in I\right\}$. If $\xi \in R \cap\left\{D_{P_{i}} \mid i \in I\right\}$ write $\xi=y / x, y$, $x \in D$. Then $y \in x R \cap D$, and $y \in x D_{P_{i}}$ for each $i$. Hence $y \in(x R \cap D) \cap\left\{x D_{P_{i}} \mid\right.$ $i \in I\}=x D$ and $\xi \in D$. Q.E.D.

2. Generalizations. We shall now generalize some of the results of $\S 1$ to the case that the $V_{i}$ are no longer assumed to be rational valuation rings but instead are merely assumed to be 1-dim quasi-local. Again we begin by sufficiently generalizing the terminology of Krull rings to fit our need. As before, $D$ and $R$ will denote domains with the same quotient field $K$. If $\mathscr{V}=\left\{V_{i}\right\}_{i \in I}$ is a set of $R$-representatives for $D, D^{\prime}(\mathscr{V})$ will denote the domain $R \cap\left(\bigcap_{i \in I} \bar{V}_{i}\right)$, which will now play an important role.

2.1. Lemma. If $\mathscr{V}=\left\{V_{i}\right\}_{i \in I}$ is a set of $R$-representatives for $D$, then $D^{\prime}(\mathscr{V})$ is almost integral over $D$ (and hence $D^{\prime}(\mathscr{V})$ is integral over $D$ if $D$ is noetherian).

Proof. If $\xi \in D^{\prime}(\mathscr{V})$, then by the $\mathrm{FC}, \xi \notin V_{i}$ for at most finitely many $i$, say $\xi \notin V_{1}, \ldots, V_{n}$. Since $\xi \in \bar{V}_{i}, \xi$ is almost integral over $V_{i}$; therefore we can choose 
$a_{i} \neq 0 \in V_{i}$, and hence in $D$, such that $a_{i} \xi^{j} \in V_{i}$ for all $j=1,2, \ldots$ Let $d=a_{1} \cdots a_{n}$, and it follows that $d \xi^{j} \in D$ for all $j=1,2, \ldots$

2.2. Lemma. If $\mathscr{V}$ is a set of $R$-representatives for $D$, then $D^{\prime}(\mathscr{V})$ is $R$-Krull and integral over $D$ if and only if $\bar{D} \cap R$ is $R$-Krull. When this occurs, then $D^{\prime}(\mathscr{V})$ $=\bar{D} \cap R$.

Proof. It suffices to show that $D^{\prime}(\mathscr{V})$ is integral over $D$ implies $D^{\prime}(\mathscr{V})=\bar{D} \cap R$ and that $\bar{D} \cap R$ is $R$-Krull implies $D^{\prime}(\mathscr{V})=\bar{D} \cap R$. We always have $D^{\prime}(\mathscr{V}) \supset \bar{D}$ $\cap R$; and if $D^{\prime}(\mathscr{V})$ is integral over $D$, the reverse inclusion is immediate. Suppose then that $\bar{D} \cap R$ is $R$-Krull. Every element of $D^{\prime}(\mathscr{V})$ is then an element of $R$ which is almost integral over $D$ by 2.1 and hence which is a fortiori almost integral over $\bar{D} \cap R$. But the rational $R$-representatives of an $R$-Krull domain are rational valuation rings and therefore are completely integrally closed, so it follows that any element of $R$ which is almost integral over $\bar{D} \cap R$ is in $\bar{D} \cap R$. Thus, $D^{\prime}(\mathscr{V})$ $\subset \bar{D} \cap R$. Q.E.D.

We call $D$ a generalized $R$-Krull domain provided there exists a set $\mathscr{V}$ of $R$ representatives for $D$ such that $D^{\prime}(\mathscr{V})$ is an $R$-Krull domain and is integral over $D$. Thus, if $R$ and $D$ are domains with quotient field $K$, then $D$ is a generalized $R$ Krull domain if there exists a set $\left\{V_{i}\right\}_{i \in I}$ of 1-dim quasi-local domains with quotient field $K$ such that $\left\{V_{i}\right\}_{i \in I}$ has FC, $R \notin \bigcap_{i \in I} V_{i}$, and $D=R \cap\left(\bigcap_{i \in I} V_{i}\right)$ and such that $D^{\prime}(\mathscr{V})=R \cap\left(\bigcap_{i \in I} \bar{V}_{i}\right)$ is $R$-Krull and integral over $D$. 2.2 shows that when $D$ is a generalized $R$-Krull domain, the domain $D^{\prime}(\mathscr{V})$, even though it is defined in terms of a set $\mathscr{V}$ of $R$-representatives for $D$, is equal to $\bar{D} \cap R$ and hence does not depend on the choice of the set $\mathscr{V}$. Therefore, in discussing a generalized $R$-Krull domain, we need only write $D^{\prime}$ rather than $D^{\prime}(\mathscr{V})$ for this domain. We remind the reader that $R$ can equal $K$ in these considerations; thus, for example, noetherian domains whose principal ideals have no imbedded components are included in our generalized $R$-Krull domains.

The difficulty in dealing with a generalized $R$-Krull domain $D$ is that one no longer has available a set of $R$-representatives with the good properties of the essential $R$-representatives of an $R$-Krull domain. (We illustrate these difficulties later in Examples 2.14 and 2.15.) However, we can single out a set of primes of $D$ which will successfully generalize the notion of essential primes of an $R$-Krull domain. Thus, we shall say that a prime ideal $P$ of the generalized $R$-Krull domain $D$ is an essential prime of $D$ if every prime of $D^{\prime}$ lying over $P$ is an essential prime of the $R$-Krull domain $D^{\prime}$. We next give some important characterizations of essential primes of a generalized $R$-Krull domain, similar to those characterizations of essential primes of an $R$-Krull domain given in 1.5. 2.4 deals with characterizations which are intrinsic in the sense that they do not involve a set of $R$ representatives, while 2.6 gives some characterizations which do involve such a set.

We need the following lemma, which will be used in localizing. 
2.3. LEMmA. Let $\mathscr{V}=\left\{V_{i}\right\}_{i \in I}$ be a set of $R$-representatives for the generalized $R$-Krull domain $D$, and let $\left\{V_{j}\right\}_{j \in J}$ be those $V_{i}$ whose center on $D$ does not intersect $S$. Then $D_{S}=R_{S} \cap\left(\bigcap_{j \in J} V_{j}\right)$ and $\left(D^{\prime}\right)_{S}=R_{S} \cap\left(\bigcap_{j \in J} \bar{V}_{j}\right)$; and hence if $D_{S} \neq R_{S}$, then $D_{S}$ is generalized $R_{S}$-Krull and $\left(D^{\prime}\right)_{S}=\left(D_{S}\right)^{\prime}$.

Proof. The first assertion follows from 1.1 and the second follows from the first and the definitions.

The following intrinsic characterizations of essential primes of a generalized $R$-Krull domain follow readily from the definitions and the properties of essential primes of an $R$-Krull domain given in $\S 1$.

2.4. Proposition. Let $D$ be a generalized $R$-Krull domain and let $P$ be a prime ideal of $D$. The following statements are equivalent:

(i) Each prime ideal $P^{\prime}$ of $D^{\prime}$ lying over $P$ is an essential prime for the $R$-Krull domain $D^{\prime}$.

(ii) $P$ is a minimal prime of $D$ and $R \notin D_{P^{\prime}}^{\prime}$ for each prime $P^{\prime}$ of $D^{\prime}$ lying over $P$.

(iii) $P$ is a minimal prime of $D$ and $R$ and $D_{P}$ have no common overring $<K$.

(iv) $P$ is a minimal prime of $D$ and no prime of $R$ lies over $P$.

(v) $P$ is a minimal prime of $D$ and $R_{P}=K$.

We now wish to relate the essential primes of the generalized $R$-Krull domain $D$ to a set $\left\{V_{i}\right\}$ of $R$-representatives for $D$. Let $P_{i}$ denote the center of $V_{i}$ on $D$. If $P$ is an essential prime of $D$, then from 2.4 and 1.2 we have $P \in\left\{P_{i}\right\}$. Hence each essential prime of $D$ is a minimal element of the $\operatorname{set}\left\{P_{i}\right\}$. To classify more specifically the essential primes of $D$ in terms of the set $\left\{P_{i}\right\}$, we will make use of the following lemma.

2.5. Lemma. Let $D$ be a domain having a set of $R$-representatives $\left\{V_{i}\right\}_{i \in I}$. Let $P_{i}$ denote the center of $V_{i}$ on $D$, let $P$ be a minimal element (with respect to inclusion) of the set $\left\{P_{i}\right\}$, and let $\left\{V_{j}\right\}_{j \in J}$ be those $V_{i}$ having center $P$ on $D$. Let $\left\{P_{\beta}^{\prime}\right\}$ be the set of contractions to $D^{\prime}=R \cap\left\{\bar{V}_{i} \mid i \in I\right\}$ of the nonzero prime ideals of the $\bar{V}_{j}, j \in J$. Then any prime ideal $P^{\prime}$ of $D^{\prime}$ lying over $P$ contains $\cap P_{\beta}^{\prime}$.

Proof. Let $x \in \bigcap P_{\beta}^{\prime}$. Then, for any $j \in J, V_{j}[x]$ is a finite $V_{j}$-module, and $x$ is in the Jacobson radical of $V_{j}[x]$. Therefore, since $V_{j}$ is 1-dim quasi-local, some power of $x$ is in the nonzero conductor of $V_{j}$ in $V_{j}[x]$, and thus is in $V_{j}$. Since $J$ is finite by the finiteness condition on $R$-representatives, there exists then a positive integer $m$ such that $x^{m} \in V_{j}$ for all $j \in J$. Therefore $x^{m} \in R \cap\left\{V_{j} \mid j \in J\right\}=D_{P}$, the equality following from 1.1. Thus, there exists $s \in D \backslash P$ such that $s x^{m} \in D \cap P_{\beta}^{\prime}=P$. If then $P^{\prime}$ is any prime of $D^{\prime}$ lying over $P$, it follows that $x \in P^{\prime}$.

2.6. Theorem. Let $D$ be a generalized $R$-Krull domain, and let $\left\{V_{i}\right\}$ be a set of $R$-representatives for $D$. Let $P_{i}$ denote the center of $V_{i}$ on $D$, and let $P$ be a minimal element (with respect to inclusion) of the set $\left\{P_{i}\right\}$. The following statements are equivalent: 
(1) $P$ is an essential prime of $D$.

(2) $R \notin D_{P^{\prime}}^{\prime}$ for each prime ideal $P^{\prime}$ of $D^{\prime}$ lying over $P$.

(3) Each $V_{i}$ centered on $P$ is such that $R$ and $V_{i}$ have no common overring $<K$.

(4) Each $V_{i}$ centered on $P$ is such that $\bar{V}_{i}$ is an intersection of finitely many essential $R$-representatives for $D^{\prime}$.

Moreover, if $P$ is an essential prime of $D$, then $D_{P}=\bigcap\left\{V_{i} \mid V_{i}\right.$ is centered on $\left.P\right\}$.

Proof. (1) $\Rightarrow(2)$ and (1) $\Rightarrow(3)$ are immediate from 2.4 .

(1) $\Rightarrow$ (4). If $P$ is essential and $V_{i}$ is centered on $P$, then each maximal ideal $M$ of $\bar{V}_{i}$ lies over $P$ in $D$ and hence lies over an essential prime $M \cap D^{\prime}$ of $D^{\prime}$. Therefore $D_{M \cap D^{\prime}}^{\prime}$ is an essential $R$-representative for $D^{\prime}$; and since it is also then a rational valuation ring, $D_{M \cap D^{\prime}}^{\prime}=\left(\bar{V}_{i}\right)_{M}$. But $\bar{V}_{i}$ is the intersection of its localizations at maximal ideals, so $\bar{V}_{i}$ is an intersection of essential $R$-representatives for $D^{\prime}$. That there are only finitely many such $R$-representatives follows from the finiteness condition on $R$-representatives, since each of these representatives is centered on $P$ in $D$.

(4) $\Rightarrow$ (3). If $R$ and $V_{i}$ have a common overring $<K$ for some $V_{i}$ centered on $P$, then they have a common valuation overring $W<K$. But $W \supset \bar{V}_{i}$; so if $\bar{V}_{i}$ is a finite intersection of rational valuation rings, then $W$ must be one of them [11, p. 38]. But then $W$ would be an essential $R$-representative for $D^{\prime}$, a contradiction to $R \subset W$.

We need the following observation for the remainder of the proof: If $P^{\prime}$ is a prime ideal of $D^{\prime}$ lying over $P$ such that $R \notin D_{P^{\prime}}^{\prime}$, then $P^{\prime}$ is an essential prime of $D^{\prime}$. For, by $1.2, P^{\prime}$ contains an essential prime $Q^{\prime}$ of $D^{\prime}$, and then $Q=Q^{\prime} \cap D \subset P$. If $Q<P$, the assumption that $P$ is minimal in the set of centers $\left\{P_{i}\right\}$ implies $\left(V_{i}\right)_{Q}=K$ for all $i$. But then $R \subset R_{Q}=D_{Q} \subset D_{Q^{\prime}}^{\prime}$, the equality following from 2.3; and this contradicts the fact that $Q^{\prime}$ is an essential prime of $D^{\prime}$. Thus, $Q=P$; and therefore $Q^{\prime}=P^{\prime}$ since $D^{\prime}$ is integral over $D$.

$(2) \Rightarrow(1)$. In view of 2.4 , it suffices to show that $P$ is a minimal prime of $D$. Since $D^{\prime}$ is integral over $D$, we need only show that each prime $P^{\prime}$ of $D^{\prime}$ lying over $P$ is minimal. By the above observation, any such $P^{\prime}$ is an essential prime of $D^{\prime}$ and hence is minimal.

(3) $\Rightarrow(1)$. Let $\left\{V_{j}\right\}_{j \in J}$ be those $V_{i}$ having center $P$ on $D$, and let $\left\{P_{\beta}^{\prime}\right\}$ denote the set of prime ideals of $D^{\prime}$ that are contractions of nonzero primes of the $\bar{V}_{j}, j \in J$. (3) assures that $R \notin D_{P_{\beta}}^{\prime}$ for each $P_{\beta}^{\prime}$; so by the above observation, each $P_{\beta}^{\prime}$ must be an essential prime of the $R$-Krull domain $D^{\prime}$. Since $D^{\prime}$ has only finitely many essential primes lying over $P,\left\{P_{\beta}^{\prime}\right\}$ is finite. By 2.5 , each prime of $D^{\prime}$ lying over $P$ must then be in the set $\left\{P_{\beta}^{\prime}\right\}$, i.e. $P$ is an essential prime of $D$.

For the final assertion of the theorem, observe that, by $2.4, P$ is minimal and $R_{P}=K$ for anv essential prime $P$ of $D$. Hence, by 2.3 ,

$$
D_{P}=\bigcap\left\{V_{i} \mid V_{i} \text { has center } P \text { on } D\right\} \text {. Q.E.D. }
$$

We are now ready to examine noetherian properties of $D, R$ and the $V_{i}$. 
2.7. THEOREM. Let $D$ be a noetherian domain having a set $\left\{V_{i}\right\}_{i \in I}$ of $R$-representatives such that $R \nsubseteq \bigcap_{i \in I} \bar{V}_{i}$. Then $D$ is a generalized $R$-Krull domain. Moreover, if $V \in\left\{V_{i}\right\}$ is centered on an essential prime of $D$, then $V$ is noetherian. Thus, for example, if for each $i, R$ and $V_{i}$ have no common overring $<K$ and there are no proper containment relations among the centers $P_{i}$ of the $V_{i}$ on $D$, then all the $V_{i}$ are noetherian.

Proof. If $D$ is noetherian, then the integral closure $\bar{D}$ of $D$ is a Krull domain [11, Theorem 33.10, p. 118]. Hence by 2.2, $D$ is a generalized $R$-Krull domain. If $V$ is centered on an essential prime $P$ of $D$, then $D_{P} \subset V \subset K$ and by $2.4, P$ is a minimal prime of $D$. Thus $V$ lies between a 1-dim noetherian domain and its quotient field. By the Krull-Akizuki theorem [11, Theorem 33.2, p. 115], $V$ is noetherian. The last assertion follows from 2.6.

2.8. TheOREM. Let $D$ be a generalized $R$-Krull domain and let $\left\{V_{i}\right\}$ be a set of $R$ representatives for $D$. If each $V_{i}$ is centered on an essential maximal ideal of $D$, then $R$ is a flat $D$-module. In this case, $D$ is noetherian if and only if $R$ and every finite intersection of the $V_{i}$ are noetherian.

Proof. Let $P$ be a prime ideal of $D$. If $R \notin D_{P}$, then by 1.2, $P$ contains and hence must equal the center of some $V_{i}$ on $D$. Hence by 2.4 , no prime of $R$ lies over $P$. Since $P$ is maximal, we have $P R=R$. Thus $R$ is a flat $D$-module [14, p. 795]. Moreover, 2.6 implies that

$$
D_{P}=\bigcap\left\{V_{i} \mid V_{i} \text { has center } P \text { on } D\right\} .
$$

Hence if $R$ and every finite intersection of the $V_{i}$ are noetherian then, by $1.8, D$ is noetherian. Conversely, $D$ noetherian implies $R$ is noetherian since $R$ is a flat $D$-module contained in the quotient field of $D$. To show that $D$ noetherian implies that every finite intersection of the $V_{i}$ is noetherian, it will suffice by the KrullAkizuki theorem to show that if $P_{1}, \ldots, P_{n}$ are essential primes of $D$, then $\bigcap_{i=1}^{n} D_{P_{i}}$ is 1-dim and noetherian. By $1.9, \bigcap_{i=1}^{n} D_{P_{i}}$ is noetherian and since each nonunit of $\bigcap_{i=1}^{n} D_{P_{i}}$ is contained in $\bigcup_{i=1}^{n} P_{i} D_{P_{i}}$ we see that the contractions of the $P_{i} D_{P_{i}}$ are the only nonzero primes of $\bigcap_{i=1}^{n} D_{P_{i}}$. Hence $\bigcap_{i=1}^{n} D_{P_{i}}$ is 1-dim. Q.E.D.

In order to apply 2.8 in showing that $R$ and the $V_{i}$ noetherian imply $D$ is noetherian, it is useful to have sufficient conditions that every finite intersection of the $V_{i}$ be noetherian. We note the following consequence of the approximation theorem for independent valuation rings.

2.9. Proposition. Let $\left\{W_{i}\right\}_{i=1}^{n}$ be a family of 1-dim quasi-local domains with quotient field $K$ such that each $\bar{W}_{i}$ is a finite intersection of valuation rings. Assume that for $i \neq j, W_{i}$ and $W_{j}$ are not dominated by a common valuation ring. Then $T=\bigcap_{i=1}^{n} W_{i}$ is 1-dim, has quotient field $K$, and if $Q_{i}$ is the center of $W_{i}$ on $T$, then the $Q_{i}$ are the only nonzero primes of $T$ and $T_{Q_{i}}=W_{i}$ for each $i$. 
Proof. The nonunits of $T$ are contained in $\bigcup_{i=1}^{n} Q_{i}$ and hence every maximal ideal is some $Q_{i}$. Thus, all assertions will follow if we show $T_{Q_{i}}=W_{i}$ for each $i$. By the approximation theorem [15b, p. 45], there exists $y \in K$ having positive value in each valuation dominating $W_{i}$ and negative value in each valuation dominating $W_{j}$ for $j \neq i$. Thus $W_{i}[y]$ is a finite $W_{i}$-module with $y$ in the Jacobson radical of $W_{i}[y]$. Hence for some positive integer $m, y^{m}$ is in the conductor of $W_{i}$ in $W_{i}[y]$ and therefore in the maximal ideal of $W_{i}$. Thus $1+y^{m}$ is a unit of $W_{i}$ having negative value in each valuation dominating $W_{j}$ for $j \neq i$. Let $x=1 /\left(1+y^{m}\right)$. Then $W_{j}[x]$ is a finite $W_{j}$-module, and for $j \neq i, x$ is contained in the Jacobson radical of $W_{j}[x]$. We conclude that for some positive integer $t, x^{t} \in\left(\bigcap_{j \neq i} Q_{j}\right) \mid Q_{i}$. Hence $\left(W_{j}\right)_{Q_{i}}=K$ for $j \neq i$. Thus by 1.1 , we have $T_{Q_{i}}=\bigcap_{j=1}^{n}\left(W_{j}\right)_{Q_{i}}=W_{i}$. Q.E.D.

2.10. Corollary. Let $\left\{W_{i}\right\}_{i=1}^{n}$ be a family of 1-dim (noetherian) local domains with quotient field $K$ such that for $i \neq j, W_{i}$ and $W_{j}$ are not dominated by a common valuation ring. Then $T=\bigcap_{i=1}^{n} W_{i}$ is a 1-dim (noetherian) semilocal domain.

2.11. Corollary. Let $D$ be a generalized R-Krull domain. Assume that there exists a family $\left\{V_{i}\right\}$ of noetherian $R$-representatives for $D$ such that each $V_{i}$ is centered on a maximal ideal of $D$. If for each $i, R$ and $V_{i}$, and $V_{j}$ and $V_{i}$, for $j \neq i$, have no common overring $<K$, then $R$ is noetherian implies $D$ is noetherian.

Proof. By 2.10 every finite intersection of the $V_{i}$ is noetherian. By 2.6 each $V_{i}$ is centered on an essential prime of $D$. Hence by $2.8, R$ is noetherian implies $D$ is noetherian.

If we restrict ourselves to the question of when $D$ is noetherian implies $R$ is noetherian, we can replace the assumption in 2.8 that each $V_{i}$ is centered on an essential prime of $D$ by an assumption only involving the $V_{i}$. We use $\left(V_{i}: \bar{V}_{i}\right)$ to denote the conductor of $V_{i}$ in $\bar{V}_{i}$, i.e. $\left(V_{i}: \bar{V}_{i}\right)=\left\{a \in V_{i} \mid a \bar{V}_{i} \subset V_{i}\right\}$. Note that if $\bar{V}_{i}$ is a finite $V_{i}$-module, then $\left(V_{i}: \bar{V}_{i}\right) \neq 0$.

2.12. Lemma. Let $D$ be a domain with a set of $R$-representatives $\left\{V_{i}\right\}_{i \in I}$. Assume that $I$ is finite, say $I=\{1, \ldots, n\}$, that each $V_{i}$ is centered on a maximal ideal of $D$ and that $C_{i}=\left(V_{i}: \bar{V}_{i}\right) \neq 0, i=1, \ldots, n$. Then $D$ is noetherian implies $R$ is noetherian.

Proof. Let $D^{\prime}=R \cap\left\{\bar{V}_{i} \mid i \in I\right\} . C=\bigcap_{i=1}^{n}\left(C_{i} \cap D\right)$ is a nonzero ideal of $D$ and $C D^{\prime} \subset D$; thus $D^{\prime}$ is a finite $D$-module and hence noetherian. In particular, $R$ is noetherian if $R \subset \bigcap_{i=1}^{n} \bar{V}_{i}$; so we may assume $R \notin \bigcap_{i=1}^{n} \bar{V}_{i}$. Then since $D$ is noetherian, $D^{\prime}$ is an $R$-Krull domain by 2.7 . Let $\mathscr{E}^{\prime}$ denote the essential $R$ representatives of $D^{\prime}$ and let $P_{i}$ denote the center of $V_{i}$ on $D$. For any $D_{P^{\prime}}^{\prime} \in \mathscr{E}^{\prime}$, $P_{i} \subset P^{\prime} \cap D$ for some $i$, by 1.2. It follows from our hypothesis that $P^{\prime} \cap D$ is maximal; and hence $P^{\prime}$ is maximal, since $D^{\prime}$ is integral over $D$. Thus, $D^{\prime}$ is noetherian implies that $R$ is noetherian by 1.10 .

2.13. THEOREM. Let $D$ be a domain having a set of $R$-representatives $\left\{V_{i}\right\}_{i \in I}$. Assume that each $V_{i}$ is centered on a maximal ideal $P_{i}$ of $D$ and that $\left(V_{i}: \bar{V}_{i}\right) \neq 0$ for all $i \in I$. Then $D$ is noetherian implies that $R$ is noetherian. 
Proof. By Cohen's theorem, it suffices to see that any prime ideal $Q_{0}$ of $R$ is finitely generated. Let $Q=Q_{0} \cap D$, and let $A$ be a finite set of generators for $Q$. If $Q \subset P_{i}$ for some $i$, then $Q$ is contained in only finitely many of the $P_{i}$, say $P_{1}, \ldots, P_{n}$; and in this case, if $S=D \backslash \bigcup_{i=1}^{n} P_{i}$, then $R_{S}$ is noetherian by 1.1 and 2.12. Thus, if $Q \subset P_{i}$ for some $i$, then we can find a finite subset $B$ of $Q_{0}$ such that $B R_{S}=Q_{0} R_{S}$. Let $C=A$ if $Q \nsubseteq P_{i}$ for all $i \in I$, and otherwise let $C=A \cup B$. Claim: $Q_{0}=C R$. It suffices to show that $Q_{0} R_{M_{0}} \subset C R_{M_{0}}$ for every maximal ideal $M_{0}$ of $R$. Let $M=M_{0} \cap D$. If $R \subset D_{M}$, then $R_{M_{0}}=D_{M}$ and hence $Q_{0} R_{M_{0}}=Q D_{M}=A D_{M} \subset C R_{M_{0}}$. If $R \notin D_{M}$, then $P_{i} \subset M$ for some $i \in I$ by 1.2 ; therefore $P_{i}=M$ since $P_{i}$ is assumed maximal. If $Q \notin M$, then $Q_{0} R_{M_{0}}=R_{M_{0}}=A R_{M_{0}}=C R_{M_{0}}$. On the other hand, if $Q \subset M=P_{i}$, then $R_{S} \subset R_{M_{0}}$; and hence $Q_{0} R_{S}=B R_{S}$, which implies $Q_{0} R_{M_{0}}=B R_{M_{0}} \subset C R_{M_{0}}$. Q.E.D.

We conclude with two examples which illustrate the difficulties that can arise in considering generalized $R$-Krull domains.

2.14. Example of a generalized $R$-Krull domain $D=R \cap V_{1} \cap V_{2}$ such that $V_{2}$ is irredundant but its center on $D$ is not minimal. Let $k_{0}<k$ be fields with $k$ algebraic over $k_{0}$, let $x$ be an indeterminate over $k$ and $y$ be an element of $k[[x]]$ of strictly positive order such that $x, y$ are algebraically independent over $k$; let $R=k[x, y]_{(x, y)}[1 / x]$; let $V_{1}$ be the $x$-adic valuation ring of $k(x, y)$; let $\bar{V}_{2}=k[[x]]$ $\cap k(x, y)\left(=k+M_{2}\right.$, where $M_{2}$ is the maximal ideal of the valuation ring $\left.\bar{V}_{2}\right)$; let $V_{2}=k_{0}+M_{2}$; and let $D=R \cap V_{1} \cap V_{2}$. Note that $k[x, y]_{(x, y)}=k+M$, where $M$ is the maximal ideal of $k[x, y]_{(x, y)}$. Moreover, $k+M \subset \bar{V}_{2}$ and $M_{2} \cap k[x, y]_{(x, y)}$ $=M$. Also, $R \cap V_{1}=k[x, y]_{(x, y)}$. Therefore $D=k[x, y]_{(x, y)} \cap V_{2}=k_{0}+M$, and $D^{\prime}=k[x, y]_{(x, y)} \cap \bar{V}_{2}=k+M$. Thus, $D^{\prime}$ is integral over $D$ and $R \notin D_{M}^{\prime}=D^{\prime}$. Therefore $D$ is generalized $R$-Krull. However, $M$ is not a minimal prime ideal of $D$ since $M$ is not a minimal prime of $D^{\prime}$. Note also that no two of the rings $R, V_{1}, V_{2}$ have a common overring $<K$.

The following example is taken from [13]. It justifies the hypothesis that $D^{\prime}$ is integral over $D$ in our results on " $R$ and the $V_{i}$ noetherian imply $D$ is noetherian".

2.15. Example of a domain $D=R \cap V$ with $R$ and $V$ noetherian, $V$ centered on $a$ maximal ideal of $D, R$ and $V$ having no common overring $<K$, and yet such that $D^{\prime}=R \cap \bar{V}$ is not integral over $D$. Hence, by 2.1, $D$ is not noetherian. Let $k$ be a field, and let $x, y$ be indeterminates over $k$; let $R=k[x, y, x / y]$ and let $V_{y}$ be the $y$-adic valuation ring of $k(x, y)$. Note that $V_{y}=k(x)+M$, where $M$ is the maximal ideal of $V_{y}$. Let $V=k(\xi)+M$, where $\xi$ is chosen by means of the following lemma.

LEMma. There exists $\xi \in k(x) \mid k$ such that $k(\xi) \cap k[x]=k$.

We shall delay proving the lemma and proceed with the example.

Let $D=R \cap V$, and let $D^{\prime}=R \cap V_{y}$. (Note that $V_{y}=\bar{V}$.) Claim: $D^{\prime}=k[x, y]$. For, the essential valuations of $k[x, y]$ are exactly the $p$-adics, where $p$ is an irreducible polynomial in $k[x, y]$. Therefore $k[x, y]=\bigcap V_{p}$, where $V_{p}$ denotes the $p$-adic valuation ring. But the only $V_{p}$ which does not contain $R$ is $V_{y}$. Therefore $R \cap V_{y} \subset \bigcap V_{p}=k[x, y]$. This proves the claim. 
Now let $P$ denote the ideal $y \cdot k[x, y]$. Then $D=R \cap V=k[x, y] \cap V=k[x, y]$ $\cap(k(\xi)+M)=(k[x]+P) \cap(k(\xi)+M)=(k[x] \cap k(\xi))+P=k+P$, and $D^{\prime}$ is certainly not integral over $D$ since both $y \cdot D^{\prime}$ and $(x, y) \cdot D^{\prime}$ lie over $P$; hence, by 2.1 , $D$ is not noetherian.

We note that $P$ is not minimal since, for example, $\{f \cdot y \cdot(x+y) \mid f \in k[x, y]\}$ is easily seen to be a prime ideal of $D$ which is $<P$. Also, $R$ is not flat over $D^{\prime}$, since $(x, y) \cdot R \neq R$ and $R \notin D_{(x, y)}^{\prime}$, and hence $R$ is a fortiori not flat over $D$.

Proof of the above lemma. Let $\xi=\left(x^{2}+1\right) / x=1 / x+x$. Then $x$ is a root of $Z^{2}$ $-\xi Z+1$, so $[k(x): k(\xi)]=2$. Let $W^{\prime}$ denote the $1 / x$-adic valuation ring of $k(x)$ and $W$ the $x$-adic valuation ring of $k(x) . W$ and $W^{\prime}$ are then conjugates over $k(\xi)$ and hence $W \cap k(\xi)=W^{\prime} \cap k(\xi)$. But

$$
k[x]=\bigcap\left\{W_{\alpha} \mid W_{\alpha} \text { is a valuation ring of } k(x) \text { over } k \text { distinct from } W^{\prime}\right\} .
$$

Hence each valuation ring of $k(\xi)$ over $k$ is dominated by some $W_{\alpha}$. Thus $k[x] \cap k(\xi)=\left(\bigcap_{\alpha} W_{\alpha}\right) \cap k(\xi)=k$.

\section{REFERENCES}

1. T. Akiba, a) Remarks on generalized rings of quotients, Proc. Japan Acad. 40 (1964), 801-806. MR 31 \#4807.

b) Remarks on generalized rings of quotients. II, J. Math. Kyoto Univ. 5 (1965), 39-44. MR 34 \#185.

2. N. Bourbaki, a) Algèbre commutative. Chap. 1: Modules plats. Chap. 2: Localisation, Actualités Sci. Indust., no. 1290, Hermann, Paris, 1961. MR 36 \#146.

b) Algèbre commutative. Chap. 3: Graduations, filtrations et topologies. Chap. 4: Idéaux premiers associés et décomposition primaire, Actualités Sci. Indust., no. 1293, Hermann, Paris, 1961. MR 30 \#2027.

c) Algèbre commutative. Chap. 5: Entièrs. Chap. 6: Valuations, Actualités Sci. Indust., no. 1308, Hermann, Paris, 1964. MR 33 \#2660.

3. P. M. Eakin, Jr., The converse to a well known theorem on Noetherian rings, Math. Ann. 177 (1968), 278-282. MR 37 \#7360.

4. P. Eakin and W. Heinzer, Non-finiteness in finite dimensional Krull domains, J. Algebra 14 (1970), 333-340.

5. R. W. Gilmer, Jr., Overrings of Prüfer domains, J. Algebra 4 (1966), 331-340. MR 34 \#2609.

6. W. Heinzer, On Krull overrings of an affine ring, Pacific J. Math. 29 (1969), 145-149. MR 39 \#5537.

7. W. Heinzer, J. Ohm and R. L. Pendleton, On integral domains of the form $\cap D_{P}, P$ minimal, J. Reine Angew. Math. 241 (1970), 147-159. MR 41 \#8393.

8. D. Lazard, a) Thesis, Paris, 1968.

b) Autour de la platitude, Bull. Soc. Math. France 97 (1969), 81-128. MR 40 \#7310.

9. K. R. Nagarajan, Groups acting on Noetherian rings, Nieuw Arch. Wisk. (3) 16 (1968), 25-29. MR 37 \#5202.

10. M. Nagata, A type of subrings of a noetherian ring, J. Math. Kyoto Univ. 8 (1968), 465-467. MR 38 \#4460.

11. - Local rings, Interscience Tracts in Pure and Appl. Math., no. 13, Interscience, New York, 1962. MR 27 \#5790.

12. J. Ohm, Some counter examples related to integral closure in $D[[x]]$, Trans. Amer. Math. Soc. 122 (1966), 321-333. MR 34 \#2613. 
13. J. Ohm and R. Pendleton, Weak Krull rings (unpublished).

14. F. Richman, Generalized quotient rings, Proc. Amer. Math. Soc. 16 (1965), 794-799. MR 31 \#5880.

15. O. Zariski and P. Samuel, Commutative algebra. Vols. I, II, University Series in Higher Math., Van Nostrand, Princeton, N. J., 1957, 1960. MR 19, 833; MR 22 \#11006.

Department of Mathematics, Purdue University, Lafayette, Indiana 47907*

Department of Mathematics, Louisiana State University, Baton Rouge, Louisiana 70803

* Current address of both authors. 